KYUNGPOOK Math. J. 53(2013), 1-12

http://dx.doi.org/10.5666/KMJ.2013.53.1.1

\title{
Subordination and Superordination for Multivalent Functions associated with the Differintegral Operator
}

OH SANG KWON

Department of Mathematics, Kyungsung University, Busan 608-736, Korea

e-mail : oskwon@ks.ac.kr

NAK Eun ChO*

Department of Applied Mathematics, Pukyong National University, Busan 608-737,

Korea

e-mail : necho@pknu.ac.kr

ABSTRACT. The purpose of the present paper is to obtain some subordination- and superordination- preserving properties for multivalent function associated the differintegral operators defined on the space of normalized analytic functions in the open unit disk. The sandwich type theorem for the integral operator is also considered.

\section{Introduction}

Let $\mathcal{H}=\mathcal{H}(\mathbb{U})$ denote the class of analytic functions in the open unit disk $\mathbb{U}=\{z \in \mathbb{C}:|z|<1\}$. For $a \in \mathbb{C}$, let

$$
\mathcal{H}[a, n]=\left\{f \in \mathcal{H}: f(z)=a+a_{n} z^{n}+a_{n+1} z^{n+1}+\cdots\right\} .
$$

We also denote $\mathcal{A}$ by the subclass of $\mathcal{H}[a, 1]$ with the usual normalization $f(0)=$ $f^{\prime}(0)-1=0$ and $f^{(p+1)}(0) \neq 0$.

Let $f$ and $F$ be members of $\mathcal{H}$. The function $f$ is said to be subordinate to $F$, or $F$ is said to be superordinate to $f$, if there exists a function $w$ analytic in $\mathbb{U}$, with $w(0)=0$ and $|w(z)|<1$, and such that $f(z)=F(w(z))$. In such a case, we write $f \prec F$ or $f(z) \prec F(z)$. If the function $F$ is univalent in $\mathbb{U}$, then we have $f \prec F$ if and only if $f(0)=F(0)$ and $f(\mathbb{U}) \subset F(\mathbb{U})$ (cf. [6]).

Definition 1.1([6]). Let $\phi: \mathbb{C}^{2} \rightarrow \mathbb{C}$ and let $h$ be univalent in $\mathbb{U}$. If $p$ is analytic in $\mathbb{U}$ and satisfies the differential subordination:

$$
\phi\left(p(z), z p^{\prime}(z)\right) \prec h(z),
$$

* Corresponding Author.

Received July 16, 2010; accepted September 14, 2012.

2010 Mathematics Subject Classification: 30C80, 30C45.

Key words and phrases: subordination, superordination, univalent function, convex function, differintegral operator. 
then $p$ is called a solution of the differential subordination. The univalent function $q$ is called a dominant of the solutions of the differential subordination, or more simply a dominant if $p \prec q$ for all $p$ satisfying (1.1). A dominant $\tilde{q}$ that satisfies $\tilde{q} \prec q$ for all dominants $q$ of (1.1) is said to be the best dominant.

Definition 1.2([7]). Let $\varphi: \mathbb{C}^{2} \rightarrow \mathbb{C}$ and let $h$ be analytic in $\mathbb{U}$. If $p$ and $\varphi\left(p(z), z p^{\prime}(z)\right)$ are univalent in $\mathbb{U}$ and satisfy the differential superordination:

$$
h(z) \prec \varphi\left(p(z), z p^{\prime}(z)\right),
$$

then $p$ is called a solution of the differential superordination. An analytic function $q$ is called a subordinant of the solutions of the differential superordination, or more simply a subordinant if $q \prec p$ for all $p$ satisfying (1.2). A univalent subordinant $\tilde{q}$ that satisfies $q \prec \tilde{q}$ for all subordinants $q$ of (1.2) is said to be the best subordinant.

Definition 1.3([6]). We denote by $Q$ the class of functions $f$ that are analytic and injective on $\overline{\mathbb{U}} \backslash E(f)$, where

$$
E(f)=\left\{\zeta \in \partial \mathbb{U}: \lim _{z \rightarrow \zeta}=\infty\right\},
$$

and are such that $f^{\prime}(\zeta) \neq 0$ for $\zeta \in \partial \mathbb{U} \backslash E(f)$.

Let $\mathcal{A}_{p}$ denote the class of functions $f$ normalized by

$$
f(z)=z^{p}+\sum_{k=1}^{\infty} a_{k+p} z^{k+p} \quad(p \in \mathbb{N}=\{1,2, \cdots\})
$$

which are analytic and $p$-valent in the open unit disk $\mathbb{U}$.

With a view to introducing a fractional differintegral operator, we begin by recalling the following definitions of fractional calculus (that is, fractional intgral and fractional derivative of an arbitrary order) considered by Owa [9] (see also [10]).

Definition 1.4. The fractional integral of order $\lambda(\lambda>0)$ is defined, for a function $f$, analytic in a simply-connected region of the complex plane containing the origin by

$$
D_{z}^{-\lambda} f(z)=\frac{1}{\Gamma(\lambda)} \int_{0}^{z} \frac{f(\zeta)}{(z-\zeta)^{1-\lambda}} d \zeta
$$

where the multiplicity of $(z-\zeta)^{\lambda-1}$ is removed by requiring $\log (z-\zeta)$ to be real when $z-\zeta>0$.

Definition 1.5. Under the Definition 1.4, the fractional derivative of $f$ of order $\lambda(\lambda \geq 0)$ is defined by

$$
D_{z}^{\lambda} f(z)=\left\{\begin{array}{cl}
\frac{1}{\Gamma(1-\lambda)} \frac{d}{d z} \int_{0}^{z} \frac{f(\zeta)}{(z-\zeta)^{\lambda}} d \zeta & (0 \leq \lambda<1) \\
\frac{d^{h}}{d z^{n}} D_{z}^{\lambda-n} f(z) & \left(n \leq \lambda<n+1 ; n \in \mathbb{N}_{0}=\mathbb{N} \cup\{0\}\right),
\end{array}\right.
$$


where the multiplicity of $(z-\zeta)^{\lambda-1}$ is removed as in Definition 1 .

We observe that, for a function $f$, given by (1.3), we have

$$
D_{z}^{\lambda} f(z)=\frac{\Gamma(p+1)}{\Gamma(p+1-\lambda)} z^{p-\lambda}+\sum_{n=1}^{\infty} \frac{\Gamma(n+p+1)}{\Gamma(n+p+1-\lambda)} a_{p+n} z^{n+p-\lambda},
$$

provided that $z \in \tilde{\mathbb{U}}$, where $\tilde{\mathbb{U}}=\mathbb{U}$ if $-\infty<\lambda \leq p$ and $\tilde{\mathbb{U}}=\mathbb{U} \backslash\{0\}$ if $p<\lambda<p+1$, and $D_{z}^{\lambda} f(z)$ is, respectively, the fractional integral of $f$ of order $-\lambda$ when $-\infty<$ $\lambda<0$ and the fractional derivative of $f$ of order $\lambda$ when $0 \leq \lambda<p+1$.

In view of (1.4), we now define the fractional differintegral operator $\Omega_{z}^{\lambda, p}$ : $\mathcal{A}_{p} \longrightarrow \mathcal{A}_{p}$ for a function $f$ of the form (1.3) and for a real number $\lambda(-\infty<\lambda<$ $p+1)$ by

$$
\begin{aligned}
\Omega_{z}^{\lambda, p} f(z) & =\frac{\Gamma(p+1-\lambda)}{\Gamma(p+1)} z^{\lambda} D_{z}^{\lambda} f(z) \\
& =z^{p}+\sum_{k=1}^{\infty} \frac{\Gamma(k+p+1) \Gamma(p+1-\lambda)}{\Gamma(p+1) \Gamma(k+p+1-\lambda)} a_{k+p} z^{k+p} .
\end{aligned}
$$

It is easily seen from (1.5) that

$$
z\left(\Omega_{z}^{\lambda, p} f(z)\right)^{\prime}=(p-\lambda) \Omega_{z}^{\lambda+1, p} f(z)+\lambda \Omega_{z}^{\lambda, p} f(z) \quad(-\infty<\lambda<p ; z \in \mathbb{U}) .
$$

We also note that

$$
\Omega_{z}^{0, p} f(z)=f(z), \quad \Omega_{z}^{1, p} f(z)=\frac{z f^{\prime}(z)}{p},
$$

and, in general

$$
\Omega_{z}^{n, p} f(z)=\frac{(p-n) z^{n} f^{(n)}(z)}{p !}(n \in \mathbb{N} ; n<p+1) .
$$

The fractional differential operator $\Omega_{z}^{\lambda, p}$ with $0 \leq \lambda<1$ was investigated by Srivastava and Aouf [13]. More recently, Srivastava and Mishra [14] obtained several interesting properties and characteristics for certain subclasses of $p$-valent analytic functions involving the differintegral operator $\Omega_{z}^{\lambda, p}$ when $-\infty<\lambda<1$. We further observe that $\Omega_{z}^{\lambda, 1}$ is the operator introduced by Owa and Srivastava [10].

Making use of the principle of subordination, Miller et al. [8] investigated some subordination theorems involving certain integral operators for analytic functions in $\mathbb{U}$ (see, also [1,11]). Moreover, Miller and Mocanu [7] considered differential superordinations, as the dual concept of differential subordinations (see also [2]). In the present paper, we obtain the subordination and superordination preserving properties of the differintegral operators $\Omega_{z}^{\lambda, p}$ defined by (1.5) with the sandwichtype theorem. 
The following lemmas will be required in our present investigation.

Lemma 1.1([4]). Suppose that the function $H: \mathbb{C}^{2} \rightarrow \mathbb{C}$ satisfies the condition:

$$
\operatorname{Re}\{H(i s, t)\} \leq 0,
$$

for all real $s$ and $t \leq-n\left(1+s^{2}\right) / 2$, where $n$ is a positive integer. If the function $p(z)=1+p_{n} z^{n}+\cdots$ is analytic in $\mathbb{U}$ and

$$
\operatorname{Re}\left\{H\left(p(z), z p^{\prime}(z)\right)\right\}>0 \quad(z \in \mathbb{U}),
$$

then $\operatorname{Re}\{\mathrm{p}(\mathrm{z})\}>0$ in $\mathbb{U}$.

Lemma 1.2([5]). Let $\beta, \gamma \in \mathbb{C}$ with $\beta \neq 0$ and let $h \in \mathcal{H}(\mathbb{U})$ with $h(0)=c$. If $\operatorname{Re}\{\beta h(z)+\gamma\}>0$ for $z \in \mathbb{U}$, then the solution of the differential equation

$$
q(z)+\frac{z q^{\prime}(z)}{\beta q(z)+\gamma}=h(z) \quad(z \in \mathbb{U} ; q(0)=c)
$$

is analytic in $\mathbb{U}$ and satisfies $\operatorname{Re}\{\beta q(z)+\gamma\}>0$ for $z \in \mathbb{U}$.

Lemma 1.3([6]). Let $p \in \mathcal{Q}$ with $p(0)=a$ and let $q(z)=a+a_{n} z^{n}+\cdots$ be analytic in $\mathbb{U}$ with $q(z) \not \equiv a$ and $n \geq 1$. If $q$ is not subordinate to $p$, then there exist points $z_{0}=r_{0} \mathrm{e}^{i \theta} \in \mathbb{U}$ and $\zeta_{0} \in \partial \mathbb{U} \backslash E(f)$, for which $q\left(\mathbb{U}_{r_{0}}\right) \subset p(\mathbb{U})$,

$$
p\left(z_{0}\right)=q\left(\zeta_{0}\right) \quad \text { and } \quad z_{0} p^{\prime}\left(z_{0}\right)=m \zeta_{0} q^{\prime}\left(\zeta_{0}\right) \quad(m \geq n) .
$$

Lemma 1.4([7]). Let $q \in \mathcal{H}[a, 1]$, let $\varphi: \mathbb{C}^{2} \rightarrow \mathbb{C}$ and set $\varphi\left(q(z), z q^{\prime}(z)\right) \equiv h(z)$. If $L(z, t)=\varphi\left(q(z), t z q^{\prime}(z)\right)$ is a subordination chain and $p \in \mathcal{H}[a, 1] \cap \mathcal{Q}$, then

$$
h(z) \prec \varphi\left(p(z), z p^{\prime}(z)\right) .
$$

implies that

$$
q(z) \prec p(z) .
$$

Furthermore, if $\varphi\left(q(z), z p^{\prime}(z)\right)=h(z)$ has a univalent solution $q \in \mathcal{Q}$, then $q$ is the best subordinant.

A function $L(z, t)$ defined on $\mathbb{U} \times[0, \infty)$ is the subordination chain (or Löwner chain) if $L(\cdot, t)$ is analytic and univalent in $\mathbb{U}$ for all $t \in[0, \infty), L(z, \cdot)$ is continuously differentiable on $[0, \infty)$ for all $z \in \mathbb{U}$ and $L(z, s) \prec L(z, t)$ for $z \in \mathbb{U}$ and $0 \leq s<t$.

Lemma 1.5([12]). The function $L(z, t)=a_{1}(t) z+\cdots$, with $a_{1}(t) \neq 0$ and $\lim _{t \rightarrow \infty}\left|a_{1}(t)\right|=\infty$, is a subordination chain if and only if

$$
\operatorname{Re}\left\{\frac{z \partial L(z, t) / \partial z}{\partial L(z, t) / \partial t}\right\}>0(z \in \mathbb{U} ; 0 \leq t<\infty) .
$$




\section{Main results}

Firstly, we begin by proving the following subordination theorem involving the Komatu integral operator $\Omega_{z}^{\lambda, p}$ defined by (1.5).

Theorem 2.1. Let $f, g \in \mathcal{A}_{p}$ and $0 \leq \alpha<1,-\infty<\lambda<p$. Suppose that

(2.1) $\operatorname{Re}\left\{1+\frac{z \phi^{\prime \prime}(z)}{\phi^{\prime}(z)}\right\}>-\delta\left(z \in \mathbb{U} ; \phi(z):=\frac{(1-\alpha) \Omega_{z}^{1+\lambda, p} g(z)+\alpha \Omega_{z}^{\lambda, p} g(z)}{z^{p}}\right)$,

where

$$
\delta=\frac{(1-\alpha)^{2}+(p-\lambda)^{2}-\left|(1-\alpha)^{2}-(p-\lambda)^{2}\right|}{4(1-\alpha)(p-\lambda)} \quad(-\infty<\lambda<p)
$$

Then the subordination:

$$
\frac{(1-\alpha) \Omega_{z}^{1+\lambda, p} f(z)+\alpha \Omega_{z}^{\lambda, p} f(z)}{z^{p}} \prec \frac{(1-\alpha) \Omega_{z}^{1+\lambda, p} g(z)+\alpha \Omega_{z}^{\lambda, p} g(z)}{z^{p}},
$$

implies that

$$
\frac{\Omega_{z}^{\lambda, p} f(z)}{z^{p}} \prec \frac{\Omega_{z}^{\lambda, p} g(z)}{z^{p}} .
$$

Moreover, the function $\Omega_{z}^{\lambda, p} g(z) / z^{p}$ is the best dominant.

Proof. Let us define the functions $F$ and $G$ by

$$
F(z):=\frac{\Omega_{z}^{\lambda, p} f(z)}{z^{p}} \text { and } G(z):=\frac{\Omega_{z}^{\lambda, p} g(z)}{z^{p}},
$$

respectively. Without loss of generality, we can assume that $G$ is analytic and univalent on $\overline{\mathbb{U}}$ and that $G^{\prime}(\zeta) \neq 0(|\zeta|=1)$. Otherwise, we replace $F$ and $G$ by $F_{r}(z)=F(r z)$ and $G_{r}(z)=G(r z)$ for $0<r<1$, respectively. Then these functions satisfy the conditions of the theorem on $\overline{\mathbb{U}}$. We can prove that $F_{r}(z) \prec G_{r}(z)$, which enables us to obtain (2.4) on letting $r \rightarrow 1$.

We first show that, if the function $q$ is defined by

$$
q(z):=1+\frac{z G^{\prime \prime}(z)}{G^{\prime}(z)} \quad(z \in \mathbb{U})
$$

then

$$
\operatorname{Re}\{q(z)\}>0 \quad(z \in \mathbb{U})
$$

Taking the logarithmic differentiation on both sides of the second equation in (2.5) and using (1.6) for $g \in \mathcal{A}$, we obtain

$$
(p-\lambda) \phi(z)=(p-\lambda) G(z)+(1-\alpha) z G^{\prime}(z)
$$


Now, by differentiating both sides of (2.7), we obtain

$$
(p-\lambda) z \phi^{\prime}(z)=(1-\alpha) z G^{\prime}(z)\left(q(z)+\frac{p-\lambda}{1-\alpha}\right),
$$

which, in conjuction with (2.7), yields the relationship:

$$
\begin{aligned}
1+\frac{z \phi^{\prime \prime}(z)}{\phi^{\prime}(z)} & =1+\frac{z G^{\prime \prime}(z)}{G^{\prime}(z)}+\frac{z q^{\prime}(z)}{q(z)+(p-\lambda) /(1-\alpha)} \\
& =q(z)+\frac{z q^{\prime}(z)}{q(z)+(p-\lambda) /(1-\alpha)} \equiv h(z) .
\end{aligned}
$$

From (2.1), we have

$$
\operatorname{Re}\left\{h(z)+\frac{(p-\lambda)}{(1-\alpha)}\right\}>0(z \in \mathbb{U}),
$$

and by using Lemma 1.2, we conclude that the differential equation (2.8) has a solution $q \in \mathcal{H}(\mathbb{U})$ with $q(0)=h(0)=1$. Let us put

$$
H(u, v)=u+\frac{v}{u+(p-\lambda) /(1-\alpha)}+\delta,
$$

where $\delta$ is given by (2.2). From (2.1), (2.8) and (2.9), we obtain

$$
\operatorname{Re}\left\{H\left(q(z), z q^{\prime}(z)\right)\right\}>0 \quad(z \in \mathbb{U}) .
$$

Now we proceed to show that $\operatorname{Re}\{H(i s, t)\} \leq 0$ for all real $s$ and $t \leq-\left(1+s^{2}\right) / 2$. From (2.8), we have

$$
\begin{aligned}
\operatorname{Re}\{H(i s, t)\} & =\operatorname{Re}\left\{i s+\frac{t}{i s+(p-\lambda) /(1-\alpha)}+\delta\right\} \\
& =\frac{t(p-\lambda) /(1-\alpha)}{|(p-\lambda) /(1-\alpha)+i s|^{2}}+\delta \\
& \leq-\frac{E_{\delta}(s)}{2|(p-\lambda) /(1-\alpha)+i s|^{2}}
\end{aligned}
$$

where

$$
E_{\delta}(s):=\left(\frac{p-\lambda}{1-\alpha}-2 \delta\right) s^{2}-\frac{p-\lambda}{1-\alpha}\left(2 \delta \frac{p-\lambda}{1-\alpha}-1\right)
$$

For $\delta$ given by (2.2), we can prove easily that the expression $E_{\delta}(s)$ given by $(2.11)$ is positive or equal to zero. Hence from (2.10), we see that $\operatorname{Re}\{H(i s, t)\} \leq 0$ for all real $s$ and $t \leq-\left(1+s^{2}\right) / 2$. Thus, by using Lemma 1.1, we conclude that $\operatorname{Re}\{q(z)\}>0$ for all $z \in \mathbb{U}$. That is, $q$ is convex in $\mathbb{U}$. 
Next, we prove that the subordination condition (2.3) implies that

$$
F(z) \prec G(z)
$$

for the functions $F$ and $G$ defined by (2.5). For this purpose, we consider the function $L(z, t)$ given by

$$
L(z, t):=G(z)+\frac{1+t}{(p-\lambda) /(1-\alpha)} z G^{\prime}(z) \quad(z \in \mathbb{U} ; 0 \leq t<\infty) .
$$

We note that

$$
\left.\frac{\partial L(z, t)}{\partial z}\right|_{z=0}=G^{\prime}(0)\left(\frac{(p-\lambda) /(1-\alpha)+1+t}{(p-\lambda) /(1-\alpha)}\right) \neq 0 \quad(0 \leq t<\infty ;-\infty<\lambda<p) .
$$

This shows that the function

$$
L(z, t)=a_{1}(t) z+\cdots
$$

satisfies the condition $a_{1}(t) \neq 0$ for all $t \in[0, \infty)$. Furthermore, we have

$$
\operatorname{Re}\left\{\frac{z \partial L(z, t) / \partial z}{\partial L(z, t) / \partial t}\right\}=\operatorname{Re}\left\{(p-\lambda) /(1-\alpha)+(1+t)\left(1+\frac{z G^{\prime \prime}(z)}{G^{\prime}(z)}\right)\right\}>0 .
$$

Therefore, by virtue of Lemma $1.5, L(z, t)$ is a subordination chain. We observe from the definition of a subordination chain that

$$
\phi(z)=G(z)+\frac{1}{(p-\lambda) /(1-\alpha)} z G^{\prime}(z)=L(z, 0)
$$

and

$$
L(z, 0) \prec L(z, t) \quad(0 \leq t<\infty)
$$

This implies that

$$
L(\zeta, t) \notin L(\mathbb{U}, 0)=\phi(\mathbb{U}) \quad(\zeta \in \partial \mathbb{U} ; 0 \leq t<\infty) .
$$

Now suppose that $F$ is not subordinate to $G$, then by Lemma 1.3, there exists points $z_{0} \in \mathbb{U}$ and $\zeta_{0} \in \partial \mathbb{U}$ such that

$$
F\left(z_{0}\right)=G\left(\zeta_{0}\right) \text { and } z_{0} F\left(z_{0}\right)=(1+t) \zeta_{0} G^{\prime}\left(\zeta_{0}\right) \quad(0 \leq t<\infty) .
$$

Hence we have

$$
\begin{aligned}
L\left(\zeta_{0}, t\right) & =G\left(\zeta_{0}\right)+\frac{1+t}{(p-\lambda) /(1-\alpha)} \zeta_{0} G^{\prime}\left(\zeta_{0}\right) \\
& =F\left(z_{0}\right)+\frac{1}{(p-\lambda) /(1-\alpha)} z_{0} F^{\prime}\left(z_{0}\right) \\
& =\frac{(1-\alpha) \Omega_{z}^{1+\lambda, p} f\left(z_{0}\right)+\alpha \Omega_{z}^{\lambda, p} f\left(z_{0}\right)}{z_{0}^{p}} \in \phi(\mathbb{U}),
\end{aligned}
$$


by virtue of the subordination condition (2.3). This contracts the above observation that $L\left(\zeta_{0}, t\right) \notin \phi(\mathbb{U})$. Therefore, the subordination condition (2.3) must imply the subordination given by (2.12). Considering $F(z)=G(z)$, we see that the function $G(z)$ is the best dominant. This evidently completes the proof of Theorem 2.1.

We next prove a dual problem of Theorem 2.1, in the sense that the subordinations are replaced by superordinations.

Theorem 2.2. Let $f, g \in \mathcal{A}_{p}$ and $0 \leq \alpha<1,-\infty<\lambda<p$. Suppose that

$$
\operatorname{Re}\left\{1+\frac{z \phi^{\prime \prime}(z)}{\phi^{\prime}(z)}\right\}>-\delta \quad\left(z \in \mathbb{U} ; \phi(z):=\frac{(1-\alpha) \Omega_{z}^{1+\lambda, p} g(z)+\alpha \Omega_{z}^{\lambda, p} g(z)}{z^{p}}\right),
$$

where $\delta$ is given by (2.1), and the function $\left((1-\alpha) \Omega_{z}^{1+\lambda, p} f(z)+\alpha \Omega_{z}^{\lambda, p} f(z)\right) / z^{p}$ is univalent in $\mathbb{U}$ and $\Omega_{z}^{\lambda, p} f(z) / z^{p} \in \mathcal{Q}$. Then the superordination:

$$
\frac{(1-\alpha) \Omega_{z}^{1+\lambda, p} g(z)+\alpha \Omega_{z}^{\lambda, p} g(z)}{z^{p}} \prec \frac{(1-\alpha) \Omega_{z}^{1+\lambda, p} f(z)+\alpha \Omega_{z}^{\lambda, p} f(z)}{z^{p}}
$$

implies that

$$
\frac{\Omega_{z}^{\lambda, p} g(z)}{z^{p}} \prec \frac{\Omega_{z}^{\lambda, p} f(z)}{z^{p}} .
$$

Moreover, the function $\Omega_{z}^{\lambda, p} g(z) / z^{p}$ is the best subordinant.

Proof. Let us define the functions $F$ and $G$, respectively, by (2.5). We first note that, if the function $q$ is defined by (2.6), by using (2.7) and (2.8), then we obtain

$$
\begin{aligned}
\phi(z) & =G(z)+\frac{1-\alpha}{p-\lambda} z G^{\prime}(z) \\
& =: \varphi\left(G(z), z G^{\prime}(z)\right) .
\end{aligned}
$$

After a simple calculation, Eq. (2.14) yields the relationship:

$$
1+\frac{z \phi^{\prime \prime}(z)}{\phi^{\prime}(z)}=q(z)+\frac{z q^{\prime}(z)}{q(z)+(p-\lambda) /(1-\alpha)} .
$$

Then by using the same method as in the proof of Theorem 2.1, we can prove that $\operatorname{Re}\{q(z)\}>0$ for all $z \in \mathbb{U}$. That is, $G$ defined by (2.5) is convex(univalent) in $\mathbb{U}$.

Next, we prove that the subordination condition (2.13) implies that

$$
G(z) \prec F(z)
$$

for the functions $F$ and $G$ defined by (2.5). Now consider the function $L(z, t)$ defined by

$$
L(z, t):=G(z)+\frac{t(1-\alpha)}{p-\lambda} z G^{\prime}(z) \quad(z \in \mathbb{U} ; 0 \leq t<\infty ;-\infty<\lambda<p) .
$$

Since $G$ is convex and $(p-\lambda) /(1-\alpha)>0$, we can prove easily that $L(z, t)$ is a subordination chain as in the proof of Theorem 2.1. Therefore according to 
Lemma 1.4, we conclude that the superordination condition (2.13) must imply the superordination given by (2.15). Furthermore, since the differential equation (2.14) has the univalent solution $G$, it is the best subordinant of the given differential superordination. Therefore we complete the proof of Theorem 2.2.

If we combine this Theorem 2.1 and Theorem 2.2, then we obtain the following sandwich-type theorem.

Theorem 2.3. Let $f, g_{k} \in \mathcal{A}_{p}(k=1,2)$ and $0 \leq \alpha<1,-\infty<\lambda<p$. Suppose that

$$
\begin{aligned}
& \operatorname{Re}\left\{1+\frac{z \phi_{k}^{\prime \prime}(z)}{\phi_{k}^{\prime}(z)}\right\} \\
& >-\delta\left(z \in \mathbb{U} ; \phi_{k}(z):=\frac{(1-\alpha) \Omega_{z}^{1+\lambda, p} g_{k}(z)+\alpha \Omega_{z}^{\lambda, p} g_{k}(z)}{z^{p}} ; k=1,2\right),
\end{aligned}
$$

where $\delta$ is given by $(2.2)$, and the function $\left((1-\alpha) \Omega_{z}^{1+\lambda, p} f(z)+\alpha \Omega_{z}^{\lambda, p} f(z)\right) / z^{p}$ is univalent in $\mathbb{U}$ and $\Omega_{z}^{\lambda, p} f(z) / z^{p} \in \mathcal{Q}$. Then the subordination relation:

$$
\begin{aligned}
\frac{(1-\alpha) \Omega_{z}^{1+\lambda, p} g_{1}(z)+\alpha \Omega_{z}^{\lambda, p} g_{1}(z)}{z^{p}} & \prec \frac{(1-\alpha) \Omega_{z}^{1+\lambda, p} f(z)+\alpha \Omega_{z}^{\lambda, p} f(z)}{z^{p}} \\
& \prec \frac{(1-\alpha) \Omega_{z}^{1+\lambda, p} g_{2}(z)+\alpha \Omega_{z}^{\lambda, p} g_{2}(z)}{z^{p}},
\end{aligned}
$$

implies that

$$
\frac{\Omega_{z}^{\lambda, p} g_{1}(z)}{z^{p}} \prec \frac{\Omega_{z}^{\lambda, p} f(z)}{z^{p}} \prec \frac{\Omega_{z}^{\lambda, p} g_{2}(z)}{z^{p}} .
$$

Moreover, the functions $\Omega_{z}^{\lambda, p} g_{1}(z) / z^{p}$ and $\Omega_{z}^{\lambda, p} g_{2}(z) / z^{p}$ are the best subordinant and the best dominant, respectively.

The assumption of Theorem 2.3, that the functions $\left((1-\alpha) \Omega_{z}^{1+\lambda, p} f(z)+\right.$ $\left.\alpha \Omega_{z}^{\lambda, p} f(z)\right) / z^{p}$ and $\Omega_{z}^{\lambda, p} f(z) / z^{p}$ need to be univalent in $\mathbb{U}$, may be replaced by another conditions in the following result.

Corollary 2.1. Let $f, g_{k} \in \mathcal{A}_{p}(k=1,2)$ and $0 \leq \alpha<1,-\infty<\lambda<p$. Suppose that the condition (2.16) is satisfied and

$$
\begin{aligned}
& \operatorname{Re}\left\{1+\frac{z \psi^{\prime \prime}(z)}{\psi^{\prime}(z)}\right\} \\
& >-\delta\left(z \in \mathbb{U} ; \psi_{k}(z):=\frac{(1-\alpha) \Omega_{z}^{1+\lambda, p} f(z)+\alpha \Omega_{z}^{\lambda, p} f(z)}{z^{p}} ; f \in \mathcal{Q}\right),
\end{aligned}
$$

where $\delta$ is given by (2.2). Then the subordination relation:

$$
\begin{aligned}
\frac{(1-\alpha) \Omega_{z}^{1+\lambda, p} g_{1}(z)+\alpha \Omega_{z}^{\lambda, p} g_{1}(z)}{z^{p}} & \prec \frac{(1-\alpha) \Omega_{z}^{1+\lambda, p} f(z)+\alpha \Omega_{z}^{\lambda, p} f(z)}{z^{p}} \\
& \prec \frac{(1-\alpha) \Omega_{z}^{1+\lambda, p} g_{2}(z)+\alpha \Omega_{z}^{\lambda, p} g_{2}(z)}{z^{p}},
\end{aligned}
$$


implies that

$$
\frac{\Omega_{z}^{\lambda, p} g_{1}(z)}{z^{p}} \prec \frac{\Omega_{z}^{\lambda, p} f(z)}{z^{p}} \prec \frac{\Omega_{z}^{\lambda, p} g_{2}(z)}{z^{p}} .
$$

Moreover, the functions $\Omega_{z}^{\lambda, p} g_{1}(z) / z^{p}$ and $\Omega_{z}^{\lambda, p} g_{2}(z) / z^{p}$ are the best subordinant and the best dominant, respectively.

Proof. In order to prove Corollary 2.1, we have to show that the condition $(2.17)$ implies the univalence of $\psi(z)$ and $F(z):=\Omega_{z}^{\lambda, p} f(z) z$. Since $0<\delta \leq 1 / 2$ from $(2.2)$ in Theorem 2.1, the condition (2.17) means that $\psi$ is a close-to-convex function in $\mathbb{U}$ (see [3]) and hence $\psi$ is univalent in $\mathbb{U}$. Furthermore, by using the same techniques as in the proof of Theorem 2.3, we can prove the convexity(univalence) of $F$ and so the details may be omitted. Therefore, by applying Theorem 2.3, we obtain Corollary 2.1.

By setting $p=1$ and $\lambda=\alpha=1 / 2$ in Theorem 2.3 , so that $\delta=1 / 2$, we deduce the following consequence of Theorem 2.3.

Corollary 2.2. Let $f, g_{k} \in \mathcal{A}_{1}(k=1,2)$. Suppose that

$$
\begin{aligned}
& \operatorname{Re}\left\{1+\frac{z \phi_{k}^{\prime \prime}(z)}{\phi_{k}^{\prime}(z)}\right\} \\
& >-\frac{1}{2}\left(z \in \mathbb{U} ; \phi_{k}(z):=\frac{(1 / 2) \Omega_{z}^{3 / 2,1} g_{k}(z)+(1 / 2) \Omega_{z}^{1 / 2,1} g_{k}(z)}{z} ; k=1,2\right),
\end{aligned}
$$

and the function $\left((1 / 2) \Omega_{z}^{3 / 2,1} g_{k}(z)+(1 / 2) \Omega_{z}^{1 / 2,1} g_{k}(z)\right) / z$ is univalent in $\mathbb{U}$ and $\Omega_{z}^{1 / 2,1} f(z) / z \in Q$. Then the subordination relation:

$$
\begin{aligned}
\frac{(1 / 2) \Omega_{z}^{3 / 2,1} g_{1}(z)+(1 / 2) \Omega_{z}^{1 / 2,1} g_{1}(z)}{z} & \prec \frac{(1 / 2) \Omega_{z}^{3 / 2,1} f(z)+(1 / 2) \Omega_{z}^{1 / 2,1} f(z)}{z} \\
& \prec \frac{(1 / 2) \Omega_{z}^{3 / 2,1} g_{2}(z)+(1 / 2) \Omega_{z}^{1 / 2,1} g_{2}(z)}{z},
\end{aligned}
$$

implies that

$$
\frac{\Omega_{z}^{1 / 2,1} g_{1}(z)}{z} \prec \frac{\Omega_{z}^{1 / 2,1} f(z)}{z} \prec \frac{\Omega_{z}^{1 / 2,1} g_{2}(z)}{z} .
$$

Moreover, the functions $\Omega_{z}^{1 / 2,1} g_{1}(z) / z$ and $\Omega_{z}^{1 / 2,1} g_{2}(z) / z$ are the best subordinant and the best dominant, respectively.

By using the same method as in the proof of Theorem 2.3, we have the following sandwich-type theorem.

Theorem 2.4. Let $f, g_{k} \in \mathcal{A}_{p}(k=1,2)$ and $0 \leq \alpha<1, \lambda<0$. Suppose that

$$
\begin{aligned}
& \operatorname{Re}\left\{1+\frac{z \phi_{k}^{\prime \prime}(z)}{\phi_{k}^{\prime}(z)}\right\} \\
& >-\delta\left(z \in \mathbb{U} ; \phi_{k}(z):=\frac{(1-\alpha) \Omega_{z}^{1+\lambda, p} g_{k}(z)+\alpha \Omega_{z}^{\lambda, p} g_{k}(z)}{z^{p-1}} ; k=1,2\right),
\end{aligned}
$$


where

$$
\delta=\frac{(1-\alpha)^{2}+(p-\lambda-1+\alpha)^{2}-\left|(1-\alpha)^{2}-(p-\lambda-1+\alpha)^{2}\right|}{4(1-\alpha)(p-\lambda-1+\alpha)},
$$

and the function $\left((1-\alpha) \Omega_{z}^{1+\lambda, p} f(z)+\alpha \Omega_{z}^{\lambda, p} f(z)\right) / z^{p-1}$ is univalent in $\mathbb{U}$ and $\Omega_{z}^{\lambda, p} f(z) / z^{p-1} \in \mathcal{Q}$. Then the subordination relation:

$$
\begin{aligned}
\frac{(1-\alpha) \Omega_{z}^{1+\lambda, p} g_{1}(z)+\alpha \Omega_{z}^{\lambda, p} g_{1}(z)}{z^{p-1}} & \prec \frac{(1-\alpha) \Omega_{z}^{1+\lambda, p} f(z)+\alpha \Omega_{z}^{\lambda, p} f(z)}{z^{p-1}} \\
& \frac{\prec(1-\alpha) \Omega_{z}^{1+\lambda, p} g_{2}(z)+\alpha \Omega_{z}^{\lambda, p} g_{2}(z)}{z^{p-1}},
\end{aligned}
$$

implies that

$$
\frac{\Omega_{z}^{\lambda, p} g_{1}(z)}{z^{p-1}} \prec \frac{\Omega_{z}^{\lambda, p} f(z)}{z^{p-1}} \prec \frac{\Omega_{z}^{\lambda, p} g_{2}(z)}{z^{p-1}} .
$$

Moreover, the functions $\Omega_{z}^{\lambda, p} g_{1}(z) / z^{p-1}$ and $\Omega_{z}^{\lambda, p} g_{2}(z) / z^{p-1}$ are the best subordinant and the best dominant, respectively.

Acknowledgements This work was supported by a Research Grant of Pukyong National University(2013 year).

\section{References}

[1] T. Bulboacă, Integral operators that preserve the subordination, Bull. Korean Math. Soc., 32(1997), 627-636.

[2] T. Bulboacă, A class of superordination-preserving integral operators, Indag. Math. N. S., 13(2002), 301-311.

[3] W. Kaplan, Close-to-convex schlicht functions, Michigan Math. J., 2(1952), 169-185.

[4] S. S. Miller and P. T. Mocanu, Differential subordinations and univalent functions, Michigan Math. J., 28(1981), 157-171.

[5] S. S. Miller and P. T. Mocanu, Univalent solutions of Briot-Bouquet differential equations, J. Different. Equations, 56(1985), 297-309.

[6] S. S. Miller and P. T. Mocanu, Differential subordination, Theory and Application, Marcel Dekker, Inc., New York, Basel, 2000.

[7] S. S. Miller and P. T. Mocanu, Subordinants of differential superordinations, Complex Var. Theory Appl., 48(2003), 815-826.

[8] S. S. Miller, P. T. Mocanu and M. O. Reade, Subordination-preserving integral operators, Trans. Amer. Math. Soc., 283(1984), 605-615.

[9] S. Owa, On the distortion theorems I, Kyungpook Math. J., 18(1978), 53-59. 
[10] S. Owa and H. M. Srivastava, Univalent and starlike generalized hypergeometric functions, Canad. J. Math., 39(1987), 1057-1077.

[11] S. Owa and H. M. Srivastava, Some subordination theorems involving a certain family of integral operators, Integral Transforms Spec. Funct., 15(2004), 445-454.

[12] Ch. Pommerenke, Univalent Functions, Vanderhoeck and Ruprecht, Göttingen, 1975.

[13] H. M. Srivastava and M. K. Aouf, A certain fractional derivative operator and its applications to a new class of analytic and multivalent functions with negative coeffcients. I and II , J. Math. anal. Appl., 171(1992), 1-13; ibid. 192(1995), 673-688.

[14] H. M. Srivastava and A. K. Mishra, A fractional differintegral operator and its applications to a nested class of multivalent functions with negative coefficients, Adv. Stud. Contemp. Math., 7(2003), 203-214. 